\title{
Methodological approaches to help unravel the intracellular metabolome of Bacillus subtilis
}

\author{
Hanna Meyer ${ }^{1}$, Hendrikje Weidmann ${ }^{2,3}$ and Michael Lalk ${ }^{1 *}$
}

\begin{abstract}
Background: Bacillus subtilis (B. subtilis) has become widely accepted as a model organism for studies on Gram-positive bacteria. A deeper insight into the physiology of this prokaryote requires advanced studies of its metabolism. To provide a reliable basis for metabolome investigations, a validated experimental protocol is needed since the quality of the analytical sample and the final data are strongly affected by the sampling steps. To ensure that the sample analyzed precisely reflects the biological condition of interest, outside biases have to be avoided during sample preparation.

Results: Procedures for sampling, quenching, extraction of metabolites, cell disruption, as well as metabolite leakage were tested and optimized for B. subtilis. In particular the energy status of the bacterial cell, characterized by the adenylate energy charge, was used to evaluate sampling accuracy. Moreover, the results of the present study demonstrate that the cultivation medium can affect the efficiency of the developed sampling procedure.

Conclusion: The final workflow presented here allows for the reproducible and reliable generation of physiological data. The method with the highest qualitative and quantitative metabolite yield was chosen, and when used together with complementary bioanalytical methods (i.e., GC-MS, LC-MS and ${ }^{1} \mathrm{H}-\mathrm{NMR}$ ) provides a solid basis to gather information on the metabolome of $B$. subtilis.
\end{abstract}

Keywords: Bacillus subtilis, Metabolomics, Sampling, Energy charge, Quenching, Filtration

\section{Background}

As an essential component of the functional genomics approach, metabolomics focuses on the quantitative and qualitative analysis of small molecules [1,2]. Of central importance in metabolomics is the development of detailed protocols for reproducible sample preparation, thus allowing for the generation of useful, physiological data [3-6]. More recently, the optimization of the analytical platform and final data analysis have been a major focus. In the past years numerous improvements for ${ }^{13} \mathrm{C}$ - and ${ }^{1} \mathrm{H}-\mathrm{NMR}$, GC-MS, GCxGC-MS, LC-MS, LC-MS/MS, as well as $\mathrm{CE} / \mathrm{MS}$, have been reported. In addition, new convenient tools for data analysis and visualization have been developed, such as mzMINE $[7,8]$ and XCMS for MS data [9]; GAVIN [10] and Metab [11] for GC-MS analysis; BATMAN [12] and BQuant [13] for NMR analysis as well as, MetAssimulo [14] and MBRole [15],

\footnotetext{
* Correspondence: lalk@uni-greifswald.de

${ }^{1}$ Institute of Biochemistry, Ernst-Moritz-Arndt-University Greifswald,

Felix-Hausdorff-Strasse 4, 17487 Greifswald, Germany

Full list of author information is available at the end of the article
}

MetExplore [16] and MSEA [17] to handle and visualize data sets of increasing size.

Sampling and pre-analysis represent the first steps in the generation of metabolome data, so validated sampling protocols are key to obtain reliable data. In order to ensure that samples truly reflect the physiological situation at the moment of sampling, the introduction of metabolic changes during pre-analysis must be avoided. Taking into account the rapid turnover of certain metabolites such as ATP, rapid sampling and quenching procedures are essential. Commonly used sampling techniques for metabolomics are: I) direct quenching [18], II) centrifugation in the cold [19] and III) fast vacuum dependent filtration [20], whereby the latter method appears to be the most useful. For the first method (I), leakage of intracellular metabolites due to cell lysis during organic solvent treatment is the major drawback [5,21,22]. Indeed, as several bacteria are disrupted by organic solvents, the direct quenching method is not applicable in these cases, and this method was not used in the present study. A considerable disadvantage of the method involving centrifugation 
in the cold (II) is a substantial time factor, and inducing physiological stress during centrifugation [23]. This method was used as "control method" in the present study. The fast filtration method (III) overcomes both drawbacks. It is fast and, as cells have already been separated from the medium during treatment with organic solvent, there is no leakage problem.

An established parameter to evaluate the sampling quality is the adenylate energy charge (EC) [23-28], which represents an index of the energy state of a cell and is defined as $(\mathrm{ATP}+1 / 2 \mathrm{ADP}) /(\mathrm{AMP}+\mathrm{ADP}+\mathrm{ATP})$. A drop in the ATP concentration and/or an increase of the AMP concentration causes a decreased EC. Since most adaptation processes are ATP depended, stress induction accompanies a decrease of ATP and therewith the EC. For growing and non-stressed cells, the EC is in the range of 0.8-0.95 [24]. In metabolomics, the EC is used to monitor the cellular stress status, where stress induction during sampling is reflected in metabolic changes. Despite the fact that the EC represent one of the most important criteria for evaluating sampling methodologies, a number of published procedures do not include this criterion. [29-31] To the best of our knowledge, no specifically developed or tested metabolome sampling methodology for the Grampositive model organism $B$. subtilis that includes the EC determination has been reported. Even though claims have been made concerning the effectiveness of various sampling methods [5,32], no data concerning modification or development of methods were presented. There is only one report [26] describing an acceptable EC of $0.79 \pm 0.04$ for $B$. subtilis in mid logarithmic growth phase. However, no specific metabolome sampling procedures were tested or described because the samples were collected according to a protocol developed for transcriptome analysis [33]. This does not facilitate the assessment of the procedure for other metabolome investigations.

In the present work, we describe the development of a reproducible methodology to obtain intracellular metabolome data for B. subtilis. For this purpose, different analytical techniques were applied in order to analyze as many chemically different metabolites as possible. Whereas amino acids, organic acids and small sugar-phosphates were analyzed by GC-MS, nucleosides, nucleotides, sugarphosphates and co-factors were determined by LC-MS analysis and extracellular metabolites via ${ }^{1} \mathrm{H}$-NMR measurements. Numerous key steps during metabolomic sample preparation and processing were critically tested, including a fast sampling step, extraction of intracellular metabolites, washing of cells, cell disruption, and leakage of metabolites during an attempted liquid nitrogen cooling step before fast vacuum dependent filtration. Because it has been reported that the EC is also influenced by the type of extraction procedure [23], different methods were compared in order to identify the best; whereby not the highest yield of metabolites but rather the highest EC was of primary importance. In addition, we were able to demonstrate that with respect to the EC the culture medium composition also influences the outcome. Finally, our methodology was optimized to achieve an EC within the physiological range and to obtain a maximal amount of extracted metabolites.

\section{Results}

\section{Cell disruption}

Rapid, efficient, and gentle cell disruption is a key prerequisite for metabolite extraction in metabolome analyses. To optimize the cell disruption by organic solution treatment $(60 \%$ ethanol) combined with a freeze-thaw cycle by liquid nitrogen, the recoveries of various, chemically diverse intracellular metabolites were compared to those obtained by using the same protocol but with an additional glass bead cell disruption step [23]. The correlation coefficient of 0.94 (Figure 1) demonstrates that both methods do not differ significantly in terms of efficiency. Moreover, the regression coefficient of 1.05 indicates that there is no need for an additional glass bead cell disruption step for $B$. subtilis metabolome analyses.

\section{Extraction of intracellular metabolites - GC-MS analysis}

Extraction solutions used in metabolome analyses should be optimized for maximal metabolite extraction efficiency and minimal artificial metabolite modification during sample preparation. To identify the most appropriate extraction solution, GC-MS and LC-MS measurements were carried out. To minimize variation between the biological samples, cell sampling was always performed during the exponential growth phase $\left(\mathrm{OD}_{600}=0.5\right)$.

By use of GC-MS, efficiencies of the following extraction solutions were compared: boiling water, boiling $60 \%(\mathrm{w} / \mathrm{v})$ ethanol, cooled $60 \%(\mathrm{w} / \mathrm{v})$ ethanol, cooled $60 \%(\mathrm{w} / \mathrm{v})$ methanol, $1 \mathrm{M}$ formic acid, 100\% acetonitrile, and a mixture of methanol, water, and chloroform (4:2:4).

The principal component analysis (PCA) shown in Figure 2 demonstrates clear differences between the extraction efficiencies of the solutions, whereby the use of boiling ethanol, cooled ethanol and cooled methanol yielded more similar results than the solution containing chloroform. Even though it is obvious that these different hydrophilic extraction solutions yield more similar outcomes compared to a hydrophobic solution, a cluster separation is possible if the PCA calculation is based exclusively on the results of these three hydrophilic extraction methods (Figure 2B).

A comparison of the metabolite amounts obtained with the different extraction solutions are presented in more detail in Figure 3. 


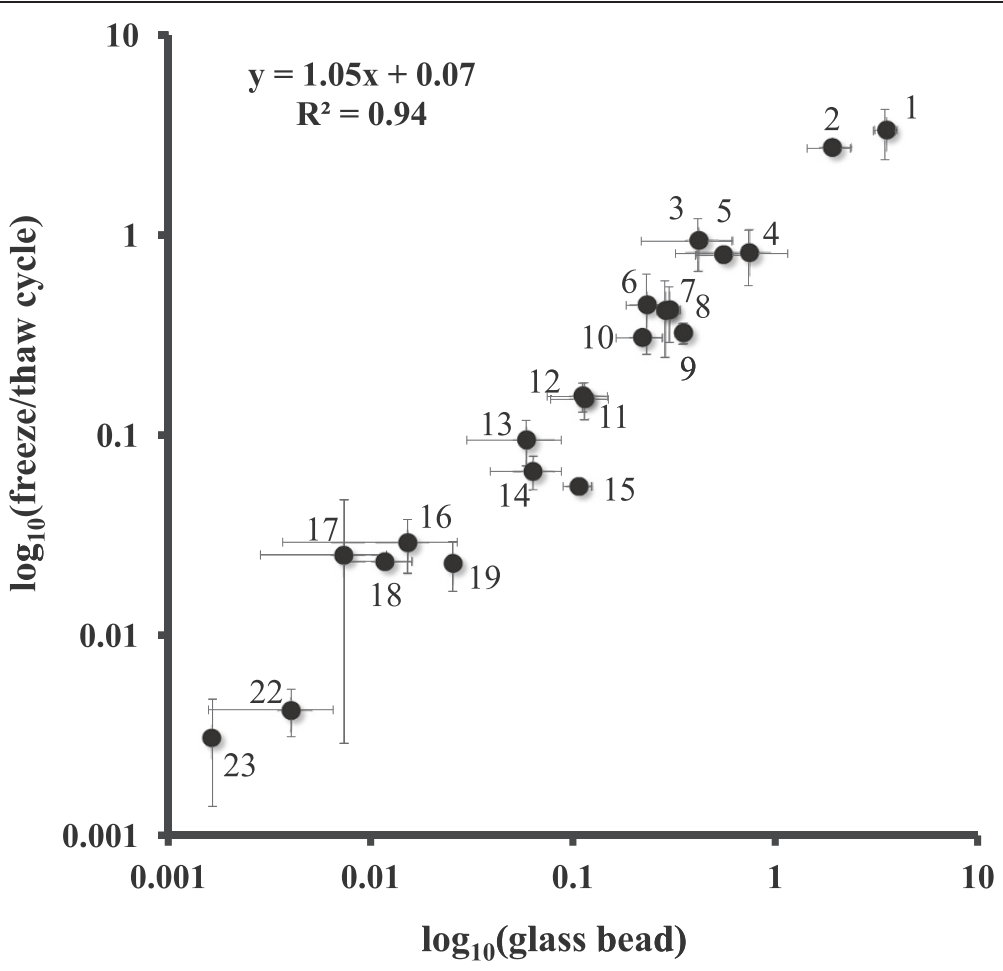

Figure 1 Metabolite correlation plot; glass bead cell disruption plotted against freeze/thaw cycle cell disruption. The correlation plot is created of the relative metabolite amounts of (1: ATP, 2: ADP, 3: AMP, 4: Fructose-1,6-bisphosphate, 5: UDP, 6:UTP, 7: GTP, 8: NADP, 9: GDP, 10: IDP, 11: UMP, 12: GMP, 13: IMP, 14: CDP, 15: FAD, 16: CMP, 17: NADPH, 18: NAD, 19: XMP, 20: NADH, 21: CoA, 22: CTP, 23: C6-sugar-phosphate). Relative metabolite amounts are calculates by the integral of the $\mathrm{m} / \mathrm{z}$ signal of each metabolite related to the $\mathrm{m} / \mathrm{z}$ internal of the internal standard Br-ATP, gained by glass bead (x-axis) cell disruption compared to freeze/thaw cycle (y-axis) cell disruption. Both axes are log ${ }_{10}$ scaled.

In the case of $1 \mathrm{M}$ formic acid, high extraction efficiency in particular for citrate, isoleucine and phosphoenolpyruvate (PEP) was achieved. Mixtures of methanol, water and chloroform successfully extracted a large set of metabolites with good efficiency. Boiling water-based extraction yielded high amounts of fumarate, citrate, sugar-phosphate(s) containing 5 carbon atoms, as well as adenine, adenosine and AMP. As described for Staphylococcus aureus, this might be caused by the fact that, aside from the sampling and quenching technique, the extraction solution also effects metabolism arrest [23]. Boiling water treatment, in contrast to organic extraction solutions, may only cause reversible inactivation of many enzymes, resulting in more pronounced hydrolysis of ATP due to residual enzyme activity that is reflected in higher amounts of lower-grade phosphorylated compounds. On the other hand, the latter could alternatively be generated by high temperature-induced ATP decomposition during the boiling-water based extraction. To investigate this further, LC-MS measurements were done to detect various nucleotides.

\section{Centrifugation-based sampling versus fast filtration sampling}

In order to find a sampling procedure which introduces the fewest metabolic changes as indicated by the EC, two methods based on either a centrifugation step or a fast vacuum-dependent filtration were compared. The latter was modified by cooling the sample in liquid nitrogen before filtration to arrest metabolism. To determine the energy charge and to evaluate the extraction efficiency of different organic solutions for nucleotides, cofactors and sugar-phosphates, an IP-LC-MS method was used.

B. subtilis cultures were harvested in exponential growth phase $\left(\mathrm{OD}_{600}=0.5\right)$ and metabolites were extracted by using $60 \%$ cold ethanol. As the centrifugation method needs at least $5 \mathrm{~min}$ even without a further washing step, results obtained with this procedure were subsequently defined as baseline and compared to those obtained by the fast vacuum filtration methods, which take maximally $30 \mathrm{sec}$ without a washing step. As expected, sampling via centrifugation resulted in an energy charge of $0.36 \pm 0.10$, which was clearly lower than the $0.43 \pm 0.10$ obtained by fast filtration with washing.

To further optimize the EC by reducing the sampling time, samples were subsequently collected without washing because the washing step during filtration is time consuming for B. subtilis cells grown in minimal medium. Indeed, omitting the washing step further increased the EC $(0.71 \pm$ 0.04). Since the cultivation medium (M9) only contained 


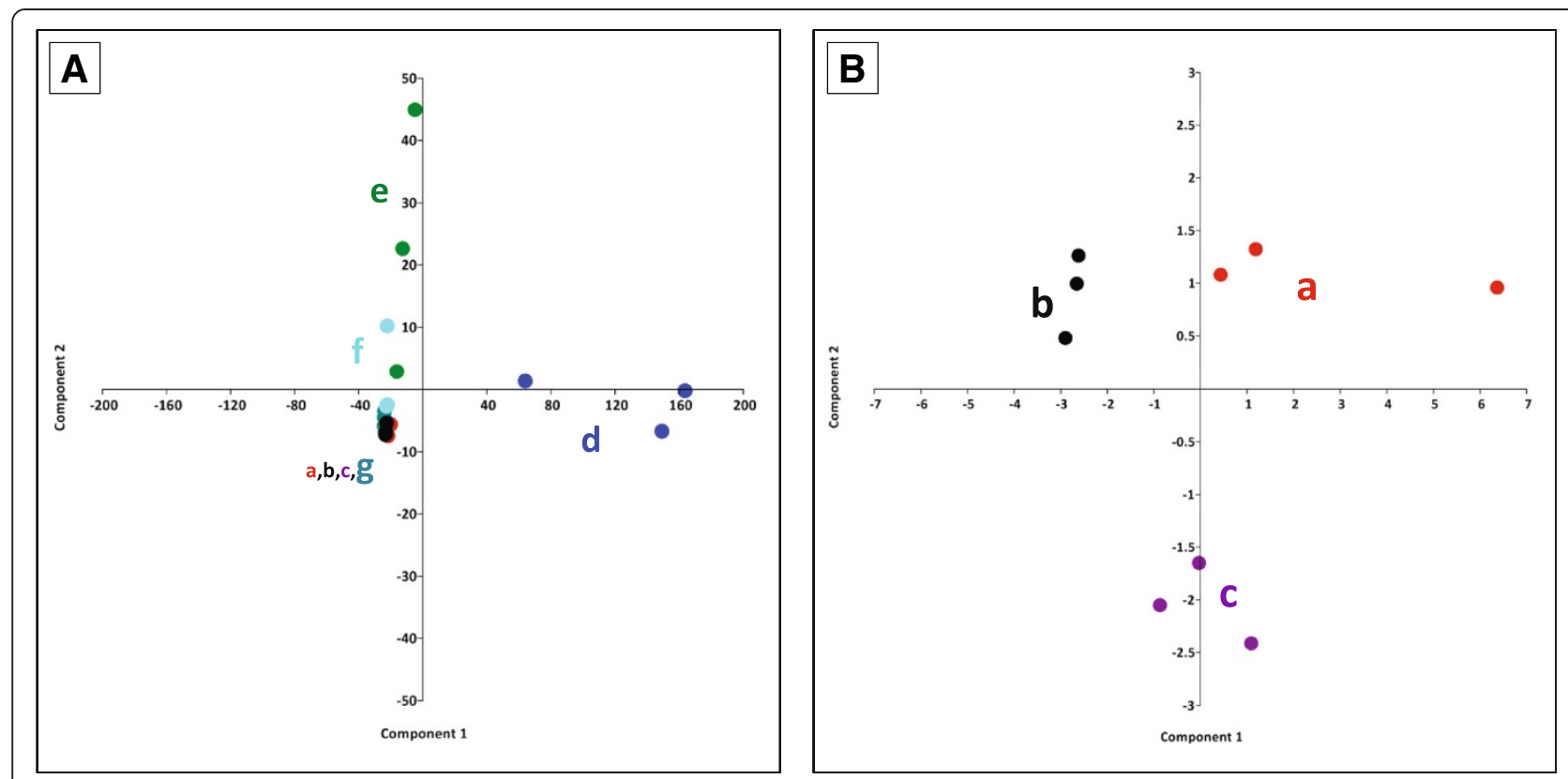

Figure 2 Principal component analysis (PCA) of the relative metabolite amounts obtained after different extraction methods. The PCA was created by PAST (Palaeontological Statistics). PAST settings were as follows: Matrix: Var-covar and Boot N: 0 .A) PCA of all GC-MS based results of the extraction efficiency tests. a (red): boiling ethanol, b (black): cooled methanol, c) (purple): cooled ethanol, d (dark blue): boiling water, e (green): methanol-chlorofom-water-mixture, $\mathrm{f}$ (cyan): acetonitrile, g (light blue): 1M formic acid (Principal component (PC)1: 93.35\% variance and PC2: 4.76\% variance) B) PCA of the GC-MS based result for the extraction power of a) boiling ethanol, b) cooled methanol, c) cooled ethanol (PC1: 63.90\% variance and PC2: $18.66 \%$ variance).

glucose and malic acid and no additional amino acids, it seemed justifiable to omit the washing step. However, it was also necessary to consider possible contamination of the measured intracellular metabolome by excreted metabolites during analysis and interpretation. Except from small amounts of valine and leucine, no amino acids were detected in the exo-metabolome by GC-MS and ${ }^{1} \mathrm{H}$-NMR analysis, while fumarate, succinate, pyruvate, 2 methylbutyric acid and acetate were clearly secreted (Figure 4). On the other hand, intracellular 2-methylbutyric acid was not detected via GC-MS, neither during exponential growth nor in the stationary phase, indicating that no significant amounts of extracellular metabolites adhere to the cells after filtration.

Although the notable EC increase that was achieved by omitting the washing step, it has been suggested that it is possible to increase the EC even further. For additional optimization of the sampling procedure, the fast vacuum-dependent filtration method was extended by a sample cooling step in liquid nitrogen immediately after cell sampling and before filtration. Indeed, introduction of the additional cooling step in liquid nitrogen resulted in a further increased EC of $0.81 \pm 0.03$, indicating improved metabolism arrest during filtration (Table 1).

During this additional cooling step, special care was taken to avoid complete freezing of the samples, since this could cause cell lysis and subsequent leakage of intracellular metabolites. To rule out significant coolinginduced cell lysis, a comparison of metabolites present in the supernatant after fast filtration sampling with or without the additional cooling step was made by GC-MS [34]. No significant differences between the metabolite profiles of both supernatants were found, where in particular the absence of detectable amounts of glutamate in both samples indicated the absence of pronounced cell lysis (Figure 5).

\section{Extraction of intracellular metabolites - LC-MS analysis}

Intracellular metabolites prepared by using cold water, cold $60 \%$ ethanol, cold $100 \%$ acetonitrile, or a mixture of methanol, water and chloroform (4:2:4) as extraction solutions, respectively, were also analyzed by LC-MS analysis. These solutions yielded the highest metabolite amounts during the GC-MS based extraction solution investigations. In contrast to the GC-MS measurements, the water-based extraction was performed with cold instead of boiling water because the boiling process was suspected of causing metabolite decomposition.

Although the cold water extraction resulted in high amounts of nearly all identifiable metabolites, the obtained EC of $0.23 \pm 0.14$ for this procedure was very low. Similarly, the methanol-water-chloroform-mixture-based extraction resulted in high abundances of the extracted metabolites, but a low EC of $0.28 \pm 0.13$. The ethanol- 


\section{$\log _{2}(\%$ to methanol extraction $)$}

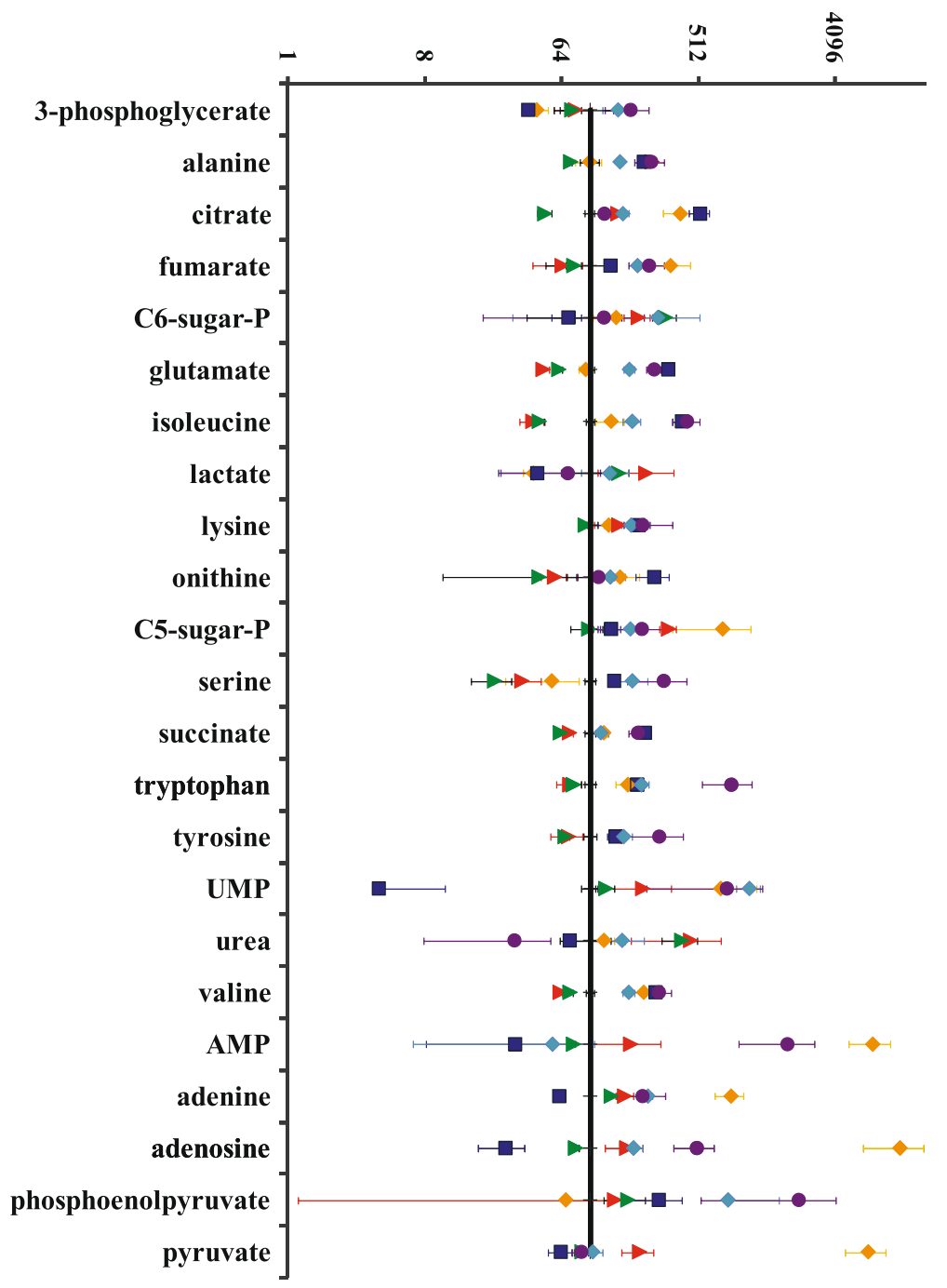

Figure 3 Comparison of the detected relative metabolite amounts via GC-MS measurement. Comparison of the detected relative metabolite amounts after several tested extraction techniques related to cooled $60 \%(\mathrm{w} / \mathrm{v})$ methanol (black line at 100\%). Boiling water (yellow), boiling (red) so as cooled (green) 60\% (w/v) ethanol, $1 \mathrm{M}$ formic acid (dark blue), 100\% acetonitrile (light blue) and methanol/water/chloroform (4:2:4) extraction (purple). Measurements were performed by GC-MS and for comparisons of the extraction potential, $60 \%$ (w/v) methanol extractions were carried out in parallel for each tested extraction solution. C5-sugar-P and C6-sugar-P refers to sugar-phosphates containing 5 or 6 carbon atoms respective. The $y$-axis is $\log _{10}$ scaled.

based extraction procedure produced a somewhat higher $\mathrm{EC}$ of $0.43 \pm 0.08$ (Table 1). Furthermore, as already observed in the GC-MS measurements, the latter procedure yielded lower extraction efficiencies (Figure 6A).

Putting these results together, we decided to perform an additional extraction step with water or chloroform after that using $60 \%$ cooled ethanol since these solutions allowed for the highest metabolite yields during both GC-MS and LC-MS measurements.

The two-step extraction procedure resulted in a higher amount of detectable intracellular metabolites compared to the extraction that used exclusively $60 \%$ cold ethanol (Figure 6B).

However, the EC still remained low for all mentioned extraction methods, underpinning the necessity of further improvement. The optimization steps demonstrated that sampling should always be carried out without washing and should use a liquid nitrogen cooling step, allowing for higher EC values (Table 1, Figure 6C). Thus, the two step extraction, first with $60 \%$ cold ethanol and second with cold water was performed after cells sampling by the extended filtration method. This resulted in 


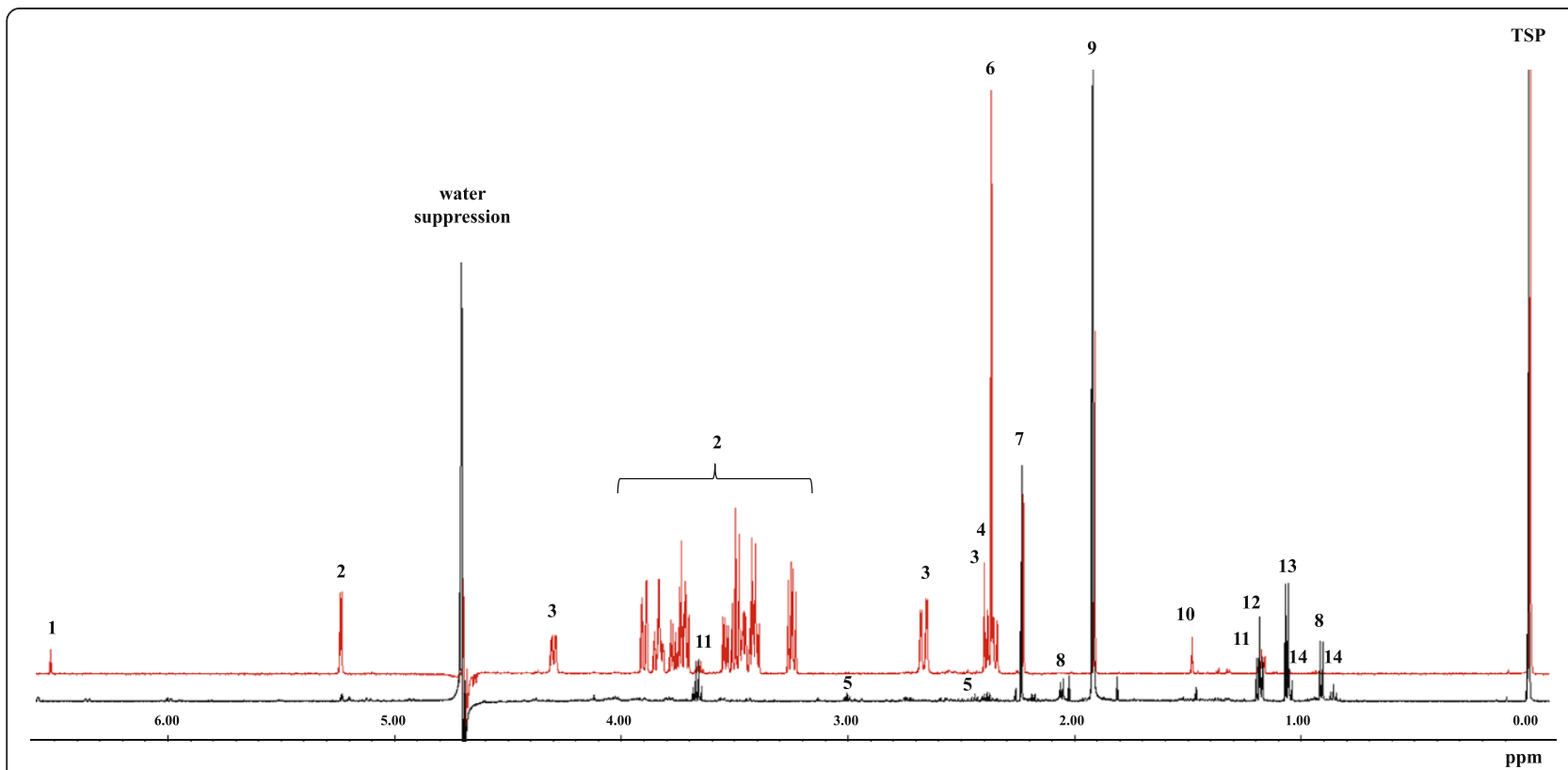

Figure 4 Extracellular metabolites obtained by ${ }^{1} \mathrm{H}-\mathrm{NMR}$ measurement (red: exponential growth and black: stationary growth). 1) fumarate, 2) glucose, 3) malate, 4) succinate, 5) 2-oxoglutarate, 6) pyruvate, 7) unknown, 8) isovaleric acid, 9) acetate, 10) 2-amino-isobutyrci acid, 11) ethanol (could be a contamination), 12) unknown, 13) isobutyric acid, 14) 2-methylbutyric acid. Further small amounts of e.g. butandiol, acetoine, valine and leucine could be detected at some time points during cell growth.

a clear increase in the EC-value to $0.81 \pm 0.03$. As demonstrated by GC-MS, the extraction with acetonitrile also resulted in high yields of metabolites. Hence, this extraction procedure was now used as second extraction step, combined with the extended filtration method and

Table 1 Overview of the energy charge obtained by the different investigated methods

\begin{tabular}{|c|c|}
\hline Sampling method & Energy charge \\
\hline $\begin{array}{l}5 \text { min centrifugation, without washing } \\
\text { (cooled } 60 \% \text { EtOH) }\end{array}$ & $0.36 \pm 0.076$ \\
\hline $\begin{array}{l}\text { Filtration, including washing } \\
\text { (cooled } \mathrm{H}_{2} \mathrm{O} \text { ) }\end{array}$ & $0.23 \pm 0.140$ \\
\hline $\begin{array}{l}\text { Filtration, including washing } \\
\text { (cooled } \mathrm{MeOH} / \mathrm{H}_{2} \mathrm{O} / \mathrm{CHCl}_{3} \text {-mixture) }\end{array}$ & $0.28 \pm 0.129$ \\
\hline $\begin{array}{l}\text { Filtration, including washing } \\
\text { (boiling } 60 \% \mathrm{EtOH} \text { ) }\end{array}$ & $0.19 \pm 0.055$ \\
\hline $\begin{array}{l}\text { Filtration, including washing } \\
\text { (cooled } 60 \% \mathrm{EtOH})\end{array}$ & $0.43 \pm 0.077$ \\
\hline $\begin{array}{l}\text { Filtration, including washing } \\
\text { (cooled } 60 \% \mathrm{EtOH} \Rightarrow \mathrm{CHCl}_{3} \text { ) }\end{array}$ & $0.41 \pm 0.069$ \\
\hline $\begin{array}{l}\text { Filtration, including washing } \\
\text { (cooled } 60 \% \mathrm{EtOH} \Rightarrow \mathrm{H}_{2} \mathrm{O} \text { ) }\end{array}$ & $0.43 \pm 0.099$ \\
\hline $\begin{array}{l}\text { Filtration, without washing } \\
\text { (cooled } 60 \% \mathrm{EtOH} \Rightarrow \mathrm{H}_{2} \mathrm{O} \text { ) }\end{array}$ & $0.71 \pm 0.040$ \\
\hline $\begin{array}{l}\mathrm{N}_{2} \text { cooling, filtration, without washing } \\
\text { (cooled } 60 \% \mathrm{EtOH} \Rightarrow \mathrm{ACN} \text { ) }\end{array}$ & $0.80 \pm 0.016$ \\
\hline $\begin{array}{l}\mathrm{N}_{2} \text { cooling, filtration, without washing } \\
\text { (cooled } 60 \% \text { EtOH } \Rightarrow \mathrm{H}_{2} \mathrm{O} \text { ) }\end{array}$ & $0.81 \pm 0.033$ \\
\hline
\end{tabular}

The used extraction solutions for each method are indicated in the brackets. liquid nitrogen cooling. The observed EC increase again confirmed that introducing a cooling step before filtration obviously causes efficient metabolism arrest (Table 1). However, the obtained amounts of intracellular metabolites were lower compared to extraction with 60\% ethanol followed by water extraction (Figure 6D). Thus, the latter extraction method was defined as the most suitable method for B. subtilis metabolome analyses.

By using this protocol, several intracellular B. subtilis metabolites were detected, including a number of amino acids and organic acids determined by GC-MS, and nucleotides, cofactors and sugar-phosphates determined by LC-MS. Moreover, analysis of LC-MS data obtained from $B$. subtilis in the stationary growth phase (retention time $10-50 \mathrm{~min}$ ) by online XCMS $[9,35]$ allowed for the detection of more than 750 molecular masses as listed in Additional file 1: Table S1. Of these, about 260 isotopes and 115 adducts were found, while about 30 of these adducts were isotope-adducts. However, only a few of these masses could be identified.

\section{Intracellular metabolite sampling after cultivation in LB media}

The protocol described above was developed for metabolome analysis of $B$. subtilis grown in chemically defined medium. However, this procedure is not suitable for B. subtilis grown in LB or other complex media, where the washing step after fast-filtration based sampling is essential for clear differentiation of intra- and extracellular 


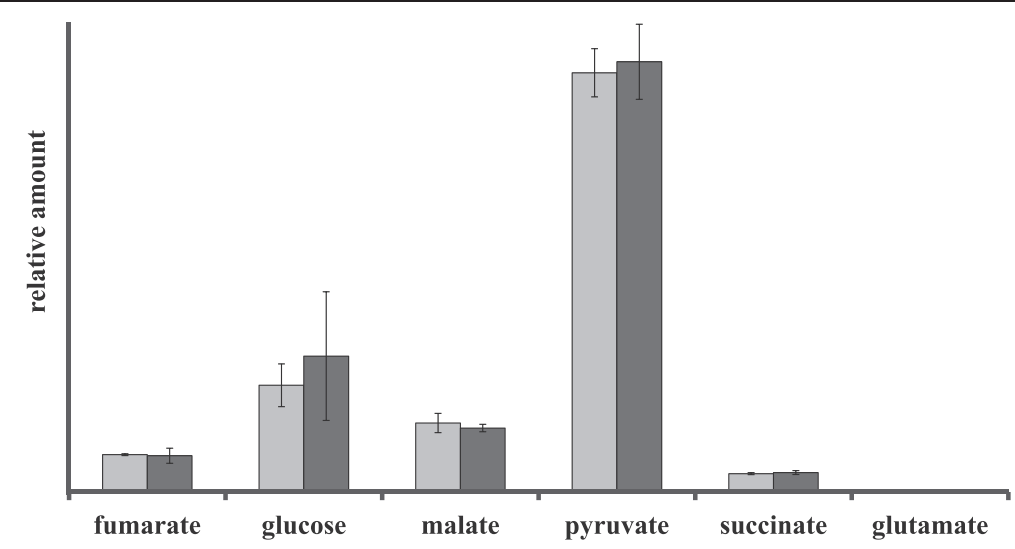

Figure 5 Cell leakage during liquid nitrogen cooling. Relative amount (related to the integral of the internal standard ribitol) of extracellular metabolites in the supernatant after direct sterile filtration compared to those in the supernatant after liquid nitrogen cooling. Light grey: direct filtration, and dark grey: after liquid nitrogen cooling.

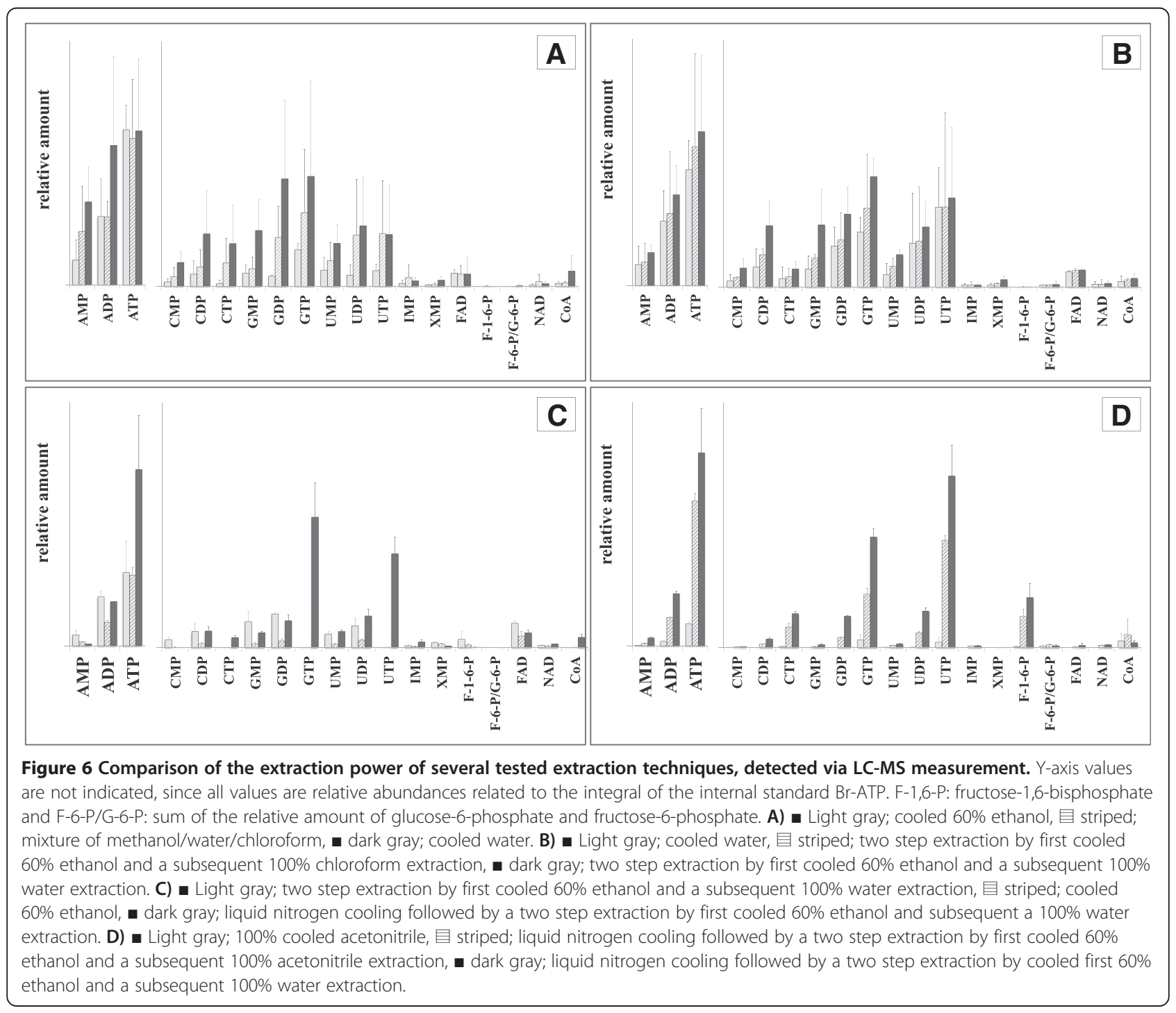


metabolites and cannot be omitted. In order to validate the usability of the method for cells grown in complex media, we cultivated $B$. subtilis in LB medium and performed sampling with and without the washing step. The obtained EC amounted to approximately 0.85 for both procedures and therefore reflected the absence of pronounced metabolic stress. This demonstrated that for cells grown in complex media, the fast-filtration based sampling protocol can be used without omitting the additional washing step.

\section{Final work flow}

In this study we optimized single workflow steps in order to produce meaningful metabolome data for B. subtilis. It became clear that common sampling methods such as cooled centrifugation and fast-vacuum dependent filtration are not applicable for metabolome analysis of B. subtilis without modifications. Therefore, fast-vacuum dependent filtration sampling was optimized by a preceding sample cooling step by using liquid nitrogen immediately after cell removal from the culture to induce metabolism arrest. After separation of the cells from the medium by fastvacuum dependent filtration, bacteria-loaded filters were directly transferred to the cold $60 \%$ ethanol extraction solution, followed by immediate freezing of the samples in liquid nitrogen. Subsequently, samples were placed on ice and cell disruption and simultaneous metabolite extraction were carried out by vortexing and shaking the sample ten times in succession. A centrifugation step separated the supernatant, which contained the extracted metabolites from cellular debris, and a second, aqueous extraction of the pellet followed. The ethanolic and aqueous supernatants were combined and stored at $-80^{\circ} \mathrm{C}$. An overview on the optimized protocol is given in Figure 7.

\section{Discussion}

This study confirms the importance of a careful assessment of a metabolome sampling methodology, customized for the specific organism of interest. The need for an optimized, tailor-made individual protocol for each analyzed microbial organism is possibly based on specific differences in the compositions of cell wall and cell membrane as well as different culture conditions. In this work, the influence of specific cultivation conditions was confirmed by the observation of clear differences between the EC obtained after washing the cells grown in minimal or in complex LB media. Aside from the necessity to develop optimized methods for each individual organism under investigation, Schaub et al. (2006) even went so far to suggest that, due to the different metabolite localizations in a cell, it seems unrealistic to use only one protocol for comprehensive metabolome analysis [36]. Even if this might be true, the available analytical techniques do not allow for taking the metabolite location in the cell into account. The newly developed method of MALDI mass spectrometry imaging may make this possible [37]. Nevertheless, the different performances of the

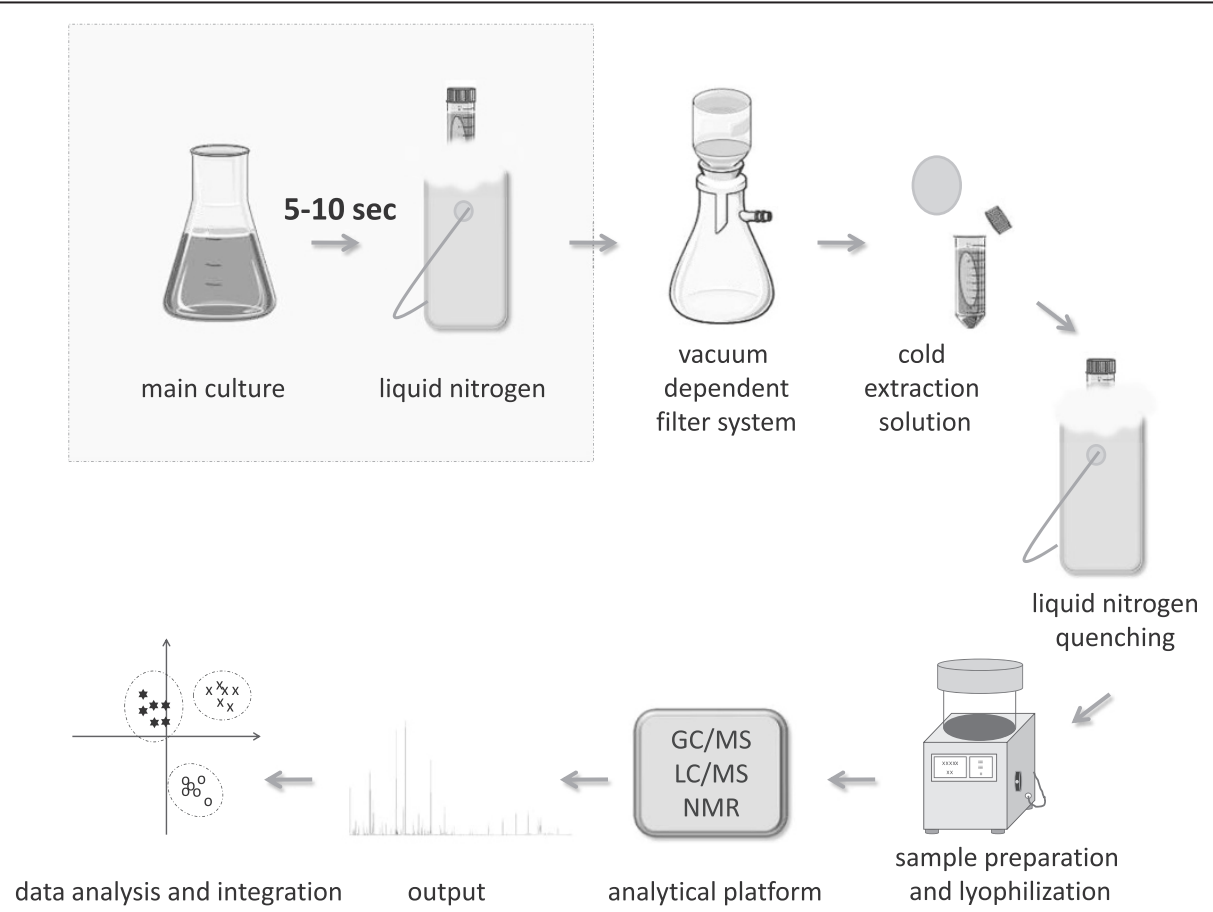

Figure 7 Workflow. Workflow for quenching, cell sampling including medium separation, cell disruption and extraction of metabolites by organic solution, lyophilization and analysis of the metabolites occurring from Bacillus subtilis cultures. 
various extraction methods could be partially caused by differences in the intracellular metabolite environments such as $\mathrm{pH}$ and more hydrophilic vs. hydrophobic milieus. Furthermore, the differencing chemical characteristics of the metabolites might influence the results of the extraction solution analyses. The PCA in Figure 2 clearly illustrates that the outcomes depend on the type of extraction solutions. These differences make it difficult to choose the most useful extraction method. In some cases the main goal is a high yield of intracellular metabolites. This might be acceptable for metabolites with a low turnover rate, such as e.g. amino acids, but for a global metabolite study that also includes short-living metabolites like ATP, the major focus should be a fast sampling technique to avoid stress during sampling. Hence, this study emphasizes the importance of the determination of the EC to evaluate the varying outcomes. Even if a given methodology results in a high amount of intracellular metabolites, this does not guarantee the generation of physiologically meaningful metabolome data with regard to metabolites with a high turnover rate. Confirming this it was demonstrated that besides the sampling and quenching procedure, the extraction solutions also influence the EC. One reason could be reversible or non-reversible enzyme inactivation, depending on the used extraction solution as assumed previously $[23,36]$. The varying outcomes for the different extraction methods shown in Figure 2, 3 and 6 further indicate that besides the metabolites AMP, ADP and ATP that influence the EC, the amounts of the other metabolites are also dependent on the sampling and extraction procedure. Investigations to study the recovery of metabolites after fastvacuum filtration followed by ethanolic extraction have been described previously by Meyer and colleagues [23]. Thus, the focus of the final workflow is primarily to obtain physiological meaningful data with an EC in the range described by Atkinson [24]. If latter criterion is guaranteed, the method that results in the highest yield of metabolites was chosen.

Even though this protocol provides the possibility for further optimized metabolome studies on B. subtilis, further challenges in metabolomics are apparent. The fact that only a fraction of the masses extracted by XCMS can be identified underlines the difficulty of metabolite identification and the need for the developed of new approaches for precise metabolite identification. Even by high resolution mass spectrometry, unambiguous metabolite identification is not always possible because some metabolites have exactly the same mass (e.g. glucose-6-phosphate and fructose-6-phosphate or succinylCoA and methyl-malonyl-CoA). Although large online databases such as the human metabolome database [38], the yeast metabolome database [39] and METLIN [40] exist, mostly the measurement of pure standard compounds is necessary for targeted metabolomics. In this context, the main problem is the limited commercial availability of some metabolites [32].

Hence, besides well-developed sampling protocols and established analytical methods the current challenge for targeted metabolomics is the identification of unknown metabolites.

\section{Conclusion}

This study describes the development of an optimized sampling methodology for B. subtilis metabolome analyses. To the best of our knowledge, this is the first B. subtilis metabolome analysis protocol that takes into account the EC. Except for work described by Coulier el al. (2006) [26], the EC for B. subtilis has not be described in the literature [32] or is below the range described by Atkinson [24]. The different outcomes seen with different extraction solutions as well as the varying results depending on the sampling protocol indicates that not only is the EC influenced by the chosen metabolome sampling protocol, but the whole metabolite profile as well. This underlines the importance of specifically developed protocols.

Finally, the described approach offers the possibility of a global view of the intracellular metabolome of B. subtilis. This would enable, for example, screening of metabolically interesting mutants or analyzing the dependency of the intracellular metabolome on growth conditions or environmental stresses.

\section{Methods}

\section{Cultivation}

A Difco sporulation medium (DSM) plate of B. subtilis $168 \operatorname{trp}^{+}[41]$ was prepared from frozen stocks $\left(-80^{\circ} \mathrm{C}\right.$, in $15 \%$ (v/v) glycerol) and incubated at $37^{\circ} \mathrm{C}$ for $24 \mathrm{~h}$. From latter plate $\mathrm{LB}$ plates were prepared and incubated at $37^{\circ} \mathrm{C}$ for $24 \mathrm{~h}$. For the pre-culture, $5 \mathrm{ml} \mathrm{LB}$ medium including $1 \mu \mathrm{g} / \mathrm{ml}$ erythromycin and $5 \mu \mathrm{g} / \mathrm{ml}$ chloramphenicol antibiotics were inoculated with a colony from the abovementioned LB plate. The cells were incubated for $4 \mathrm{~h}$ at $37^{\circ} \mathrm{C}$ and $240 \mathrm{rpm}$. The LB cultures were used to inoculate five different diluted (1:25000, 1:30000, 1: 35000, $1: 40000$ and 1:50000) overnight culture in chemical defined M9 medium containing $0.1 \%$ glucose and $0.1 \%$ malic acid. The overnight cultures were incubated for $14 \mathrm{~h}$ at $37^{\circ} \mathrm{C}$ and $300 \mathrm{rpm}$. Before inoculation of the main culture to an $\mathrm{OD}_{600 \mathrm{~nm}}$ of 0.05 , the overnight culture was tested to ensure it was in exponential growth phase $\left(\mathrm{OD}_{600 \mathrm{~nm}}=0.4-0.8\right)$. The main culture was incubated in M9 medium containing $0.1 \%$ glucose and $0.1 \%$ malic acid in a shake flask under aerobic conditions at $37^{\circ} \mathrm{C}$ and $300 \mathrm{rpm}$.

Cultivations in LB (lysogenic broth) medium were carried out as described above for M9 medium cultivation, whereas overnight culture and the main culture were incubated in LB medium. 
Intracellular metabolite sampling

Additional file 2: Table S2 (additional information) presents a list of all investigated sampling, extraction and cell disruption methods.

\section{Centrifugation sampling}

$20 \mathrm{ml}$ of the main culture was harvested in exponential growth phase $\left(\mathrm{OD}_{600}=0.5\right)$ and centrifuged for $5 \mathrm{~min}$ at $13000 \mathrm{rpm}$ and $0^{\circ} \mathrm{C}$. Afterwards the supernatant was discarded and $5 \mathrm{ml}$ of cold $60 \%$ ethanol (w/v) including $100 \mathrm{nmol}$ brom-adenosin-5'-triphosphat (Br-ATP) as internal standard was added to the cell pellet.

\section{Filtration sampling}

$20 \mathrm{ml}$ of the main culture was harvested in exponential growth phase $\left(\mathrm{OD}_{600}=0.5\right)$ via fast vacuum-dependent filtration, modified after [23]. The upper part of the filtration system was cooled at $-20^{\circ} \mathrm{C}$ for $10 \mathrm{~min}$ before sampling. The concentration of the isotonic washing solution was changed to $0.8 \% \mathrm{NaCl}$ for adaption to the growth conditions for B. subtilis grown in M9 medium (for all samples in which the washing step was carried out). After LB cultivations a $0.9 \% \mathrm{NaCl}$ solution was used for the washing step. Furthermore a liquid nitrogen pre-cooling step was appended to selected samples in order to increase the energy charge. For this purpose $20 \mathrm{ml}$ of the main culture was poured into a $50 \mathrm{ml}$ plastic centrifuge tube and cooled with liquid nitrogen for $10 \mathrm{sec}$ to stop enzymatic activity and prevent the turnover of metabolites (sample temperature after cooling $9 \pm 2^{\circ} \mathrm{C}$ ). The plastic centrifuge tube was dipped periodically (ten times and max. $10 \mathrm{sec}$ in total) in and out of the liquid nitrogen by stages and shaken carefully in between in order to avoid freezing of the sample and consequent metabolite leakage caused by cell lysis. Subsequently the cooled culture was filtered and following the filter including the cells was added to the pre-cooled $\left(30 \mathrm{~min}\right.$ at $\left.-20^{\circ} \mathrm{C}\right)$ extraction solution. Immediately after, metabolism was quenched by liquid nitrogen. The frozen samples were stored at $-80^{\circ} \mathrm{C}$ until further treatment.

\section{Extracellular metabolite sampling}

$2 \mathrm{ml}$ cell culture was sterile filtered (ø pore $0.45 \mu \mathrm{m}$, Filtropur $S^{\oplus}$, Sarstedt) rapidly into a $2 \mathrm{ml}$ tube and stored at $-20^{\circ} \mathrm{C}$ as described previously [42].

\section{Metabolite extraction}

In this work, eleven methods for metabolite extraction were investigated as listed in Table 2.

GC-MS analyses were preformed for extraction solution efficiency investigations with regard to amino acids, organic acids, small sugar phosphates and nucleoside monophosphate. IP-LC-MS analyses were mainly preformed to evaluate the energy charge of samples
Table 2 All investigated extraction solution and the downstream analytic used are noted for each extraction method

\begin{tabular}{|c|c|}
\hline Extraction solution & Applied method \\
\hline $\begin{array}{l}\text { Cooled methanol, water and chloroform } \\
\text { mixture }(4: 2: 4)\end{array}$ & GC-MS and LC-MS \\
\hline Cooled water, $100 \%$ & LC-MS \\
\hline $\begin{array}{l}\text { Boiling water, } 100 \% \text { : the filter including } \\
\text { cells was added to a plastic }\end{array}$ & GC-MS \\
\hline \multicolumn{2}{|l|}{$\begin{array}{l}\text { Centrifuge tube and the sample were } \\
\text { heated for } 10 \mathrm{~min} \text { in a } 100^{\circ} \mathrm{C} \text { water bath. }\end{array}$} \\
\hline Cooled $60 \%(w / v)$ methanol & GC-MS \\
\hline Cooled $60 \%(w / v)$ ethanol & GC-MS and LC-MS \\
\hline $\begin{array}{l}\text { Boiling } 60 \%(\mathrm{w} / \mathrm{v}) \text { ethanol, accomplished } \\
\text { as for boiling water. }\end{array}$ & GC-MS and LC-MS \\
\hline Cooled $1 \mathrm{M}$ formic acid & GC-MS \\
\hline Cooled $100 \%$ acetonitrile & GC-MS and LC-MS \\
\hline $\begin{array}{l}\text { Cooled ethanol } 60 \%(\mathrm{w} / \mathrm{v}) \text { followed by a } \\
\text { second water extraction }\end{array}$ & LC-MS \\
\hline $\begin{array}{l}\text { Cooled ethanol } 60 \%(\mathrm{w} / \mathrm{v}) \text { followed by a } \\
\text { second chloroform extraction }\end{array}$ & LC-MS \\
\hline $\begin{array}{l}\text { Cooled ethanol } 60 \%(w / v) \text { followed by a } \\
\text { second acetonitrile extraction }\end{array}$ & LC-MS \\
\hline
\end{tabular}

depending on the applied sampling and extraction method and to investigate the quantity of the cell disruption method. Moreover the IP-LC-MS data were used to evaluate the extraction efficiency of nucleotides, fructose-1,6-bis-phosphate, frucose-6-phosphate/glucose-6phosphate, FAD, NAD and CoA.

For each extraction method, $5 \mathrm{ml}$ of the extraction solution including the internal standard (100 nmol Br-ATP for LC-MS and $20 \mathrm{nmol}$ ribitol and norvaline for GCMS analysis) was added to a $50 \mathrm{ml}$ plastic centrifuge tube. The cold extraction solutions were pre-cooled at $-20^{\circ} \mathrm{C}$ for $30 \mathrm{~min}$ (water was pre-cooled at $4^{\circ} \mathrm{C}$ for $30 \mathrm{~min})$. During the sampling procedure, the solutions were kept on ice $\left(\leq 6^{\circ} \mathrm{C}\right)$. Each experiment was performed in triplicate. For comparison of the extraction methods, all samples were taken at an $\mathrm{OD}_{600 \mathrm{~nm}}$ of 0.5 and the methanol extraction was carried out in parallel for all GC-MS experiments.

\section{Cell disruption - glass bead method}

The glass bead cell disruption method was carried out as described by Meyer et al. [23]. Samples were thawed on ice $\left(\leq 6^{\circ} \mathrm{C}\right)$ and splitted to $5 \times 1 \mathrm{ml}$. Each $1 \mathrm{ml}$ extraction solution containing the cell sample was added to screw cap micro tube (SARSTEDT) filled with $0.5 \mathrm{ml}$ glass beads (Sartorius AG, diameter 0.10-0.11 mm).

Next the cells were disrupted in a homogenizer (Precellys 24) using 2 cycles for $3 \mathrm{~s}$ at $6800 \mathrm{rpm}$. 
The glass beads and the cell debris were separated from the supernatant by centrifugation for $5 \mathrm{~min}$ at $4^{\circ} \mathrm{C}$ and $13000 \mathrm{rpm}$, the aliquots were combined, and the glass beads were washed once with double-distilled water. The washing solutions were added to the metabolite extracts. The metabolite containing samples were restocked with double-distilled water to a final organic solution concentration of $10 \%$ and stored at $-80^{\circ} \mathrm{C}$ prior to lyophilization.

\section{Cell disruption - freeze/thaw cycle}

Cells disruption was carried out by the extraction solution and a freeze/thaw cycle after filtration. For this method, the $\mathrm{N}_{2}$ frozen and at $-80^{\circ} \mathrm{C}$ stored samples were thawed on ice $\left(\leq 6^{\circ} \mathrm{C}\right)$, vortexed and shaken 10 times alternately and centrifuged for $5 \mathrm{~min}$ at $4^{\circ} \mathrm{C}$ and $13000 \mathrm{rpm}$. In case of the two step extraction methods, the supernatant were filled to a new $50 \mathrm{ml}$ plastic centrifuge tube. The pellet was once more extracted by the second extraction solution (water, chloroform or acetonitrile), vortexed and shaken 10 times alternately and centrifuged for $5 \mathrm{~min}$ at $4^{\circ} \mathrm{C}$ and $13000 \mathrm{rpm}$. The supernatants were combined and the pellet was discarded.

After metabolite extraction and cell disruption, the supernatant including the extracted metabolites were restocked with double-distilled water to a final organic solution concentration of $10 \%$ and stored at $-80^{\circ} \mathrm{C}$ prior to lyophilization. All steps were carried out on ice $\left(\leq 6^{\circ} \mathrm{C}\right)$.

\section{Analytical methods \\ NMR}

$400 \mu \mathrm{L}$ of the sterile filtered (ø pore $0.45 \mu \mathrm{m}$, Filtropur $\mathrm{S}^{\oplus}$, Sarstedt) extracellular metabolite sample was buffered to pH 7.0 by addition of $200 \mu \mathrm{L}$ of a sodium hydrogen phosphate buffer (0.2 $\mathrm{mM}$ [pH 7.0], including $1 \mathrm{mM}$ TSP)) made up with $50 \% \mathrm{D}_{2} \mathrm{O}$ to provide a nuclear magnetic resonance (NMR)-lock signal. The metabolite containing samples were measured by a 600.27 MHz NMR at $310 \mathrm{~K}$ using a Bruker AVANCE-II 600 NMR spectrometer (Bruker Biospin GmbH, Rheinstetten, Germany). A modified 1D-NOESY pulse sequence was adopted and a total of 64 free induction decays (FID scans) were collected using a spectral width of $30 \mathrm{ppm}$ for a one-dimensional spectrum. AMIX 3.9 was used for data processing and analysis.

Intracellular metabolites were measured by GC-MS and LC-MS. Prior to analysis samples were lyophilized at 0.54 mbar and $-54^{\circ} \mathrm{C}$ (Christ Alpha 1-4).

\section{GC-MS (ES, quadrupol)}

GC-MS analysis was performed for amino acids, organic acids, fatty acids, sugars, nucleobases, intermediates from the glycolysis and some nucleoside monophosphates. Completely lyophilized samples were derivatized for 90 min at $37^{\circ} \mathrm{C}$ with O-methylhydroxylamin-hydrochlorid (MeOX) and $30 \mathrm{~min}$ at $37^{\circ} \mathrm{C}$ with N-methyl-N(trimethylsilyl)-trifluoroacetamid (MSTFA) and centrifuged for $2 \mathrm{~min}$ at room temperature. The supernatants were transferred into glass-vials prior to GC-MS analyses as described previously [43]. Data analysis was performed by MetaQuant [44]. For determination of the relative metabolite amounts, the mass spectrometric base peak of each metabolite was integrated and normalized to the base peak integral of the internal standard ribitol.

\section{IP-LC-MS (ESI-TOF)}

The detection of nucleotides, nucleosides, sugar-phosphates and cofactors was performed by an ion-pairing-LC-MS (IP-LC-MS) method as described earlier [45] (Agilent HPLC System 1100; Agilent Technologies, USA). Completely dried samples were dissolved in $100 \mu \mathrm{l}$ doubledistilled water and centrifuged for $2 \mathrm{~min}\left(13.000 \mathrm{rpm}, 2^{\circ} \mathrm{C}\right)$. The supernatants were transferred into glass vials with micro inserts for small volume injections.

Chromatographic separation was performed using a RP-C ${ }_{18}$ Waters $^{\oplus}$ Symmetry-Shield column (150x4.6 mm, $3.5 \mu \mathrm{m}$ ) with a $\mathrm{C}_{18}$ waters $^{\circledast}$ precolumn. The gradient flow rate was $0.3 \mathrm{ml} / \mathrm{min}$. The solutions were as follows:

A) $5 \%$ methanol and $95 \%$ water, containing $10 \mathrm{mM}$ tributylamine as the ion-pairing (IP) reagent and $15 \mathrm{mM}$ acetic acid. $\mathrm{NH}_{3}$ were used for $\mathrm{pH}$ adjustment to $\mathrm{pH} 4.9$.

B) $100 \%$ methanol.

The HPLC gradient was as described in [45].

The HPLC was coupled to a micrOTOF mass spectrometer (Bruker Daltonics, Bremen, Germany) operating in ESI negative ionization mode using a mass range from 100 to $2000 \mathrm{~m} / \mathrm{z}$.

Data analysis was carried out by QuantAnalysis ${ }^{\oplus}$ (Bruker Daltonics, Bremen, Germany). Relative metabolite amounts were calculated by the $\mathrm{m} / \mathrm{z}$ integral of each metabolite normalized to the $\mathrm{m} / \mathrm{z}$ integral of the internal standard Br-ATP.

\section{Energy charge determination}

For energy charge determination AMP, ADP and ATP have been quantified. The peak areas of the exact $\mathrm{m} / \mathrm{z}$ of latter metabolites were integrated by QuantAnalysis ${ }^{\circledR}$ (Bruker Daltonik, Bremen, Germany). The peak areas of each extracted ion $(\mathrm{m} / \mathrm{z})$ were normalized to the integral of the $\mathrm{m} / \mathrm{z}$ area of the internal standard Br-ATP. For determination of the calibration equation, different concentrations of pure standards were measured and analyzed in the same manner. Linear regression equations were determined in excel $^{-}$. The EC was calculated by the formula:

$$
\frac{[A T P]+\frac{1}{2}[A D P]}{[A T P]+[A D P]+[A M P]}
$$




\section{Additional files}

Additional file 1: Table S1. Mass list obtained by online XCMS.

Additional file 2: Table S2. List of all investigated sampling, cell disruption, leakage and extraction methods.

\section{Competing interest}

The authors declare that they have no competing interests.

\section{Authors' contributions}

HM elaborated experiments, performed cultivations, all sampling optimization steps, carried out NMR, GC-MS and LC-MS analysis, implemented data analyses and wrote the manuscript. HW performed and optimized cultivation conditions to enable further experiments. ML supervised the experiments and participated in the manuscript draft. All authors read and approved the final manuscript.

\section{Acknowledgement}

We thank Patrick J. Bednarski, Georg Homuth and Tiffany Southall for critical reading of the manuscript and Uwe Völker for helpful discussions. Further we thank Baltic Analytics for laboratory use. This work was supported by the Bundesministerium für Bildung und Forschung (BMBF, BACELL-SysMo 031397A) and the DFG (SFB/TRR34).

\section{Author details}

${ }^{1}$ Institute of Biochemistry, Ernst-Moritz-Arndt-University Greifswald, Felix-Hausdorff-Strasse 4, 17487 Greifswald, Germany. ${ }^{2}$ Interfaculty Institute for Genetics and Functional Genomics, Department of Functional Genomics, University Medicine Greifswald, Ernst-Moritz-Arndt-University Greifswald, Friedrich-Ludwig-Jahn-Str. 15a, 17489 Greifswald, Germany. ${ }^{3}$ Current address: Oncotest GmbH, Am Flughafen 12-14, Freiburg 79108, Baden-Württemberg, Germany.

Received: 6 December 2012 Accepted: 1 July 2013

Published: 11 July 2013

\section{References}

1. Koek MM, Muilwijk B, van der-Werf MJ, Hankemeier T: Microbial metabolomics with gas chromatography/mass spectrometry. Anal Chem 2006, 78:1272-1281.

2. Fiehn O: Metabolomics - the link between genotypes and phenotypes. Plant Mol Biol 2002, 48:155-171.

3. Villas-Boas SG, Hojer-Pedersen J, Akesson M, Smedsgaard J, Nielsen J: Global metabolite analysis of yeast: evaluation of sample preparation methods. Yeast 2005, 22:1155-1169.

4. Spura J, Reimer LC, Wieloch P, Schreiber K, Buchinger S, Schomburg D: A method for enzyme quenching in microbial metabolome analysis successfully applied to gram-positive and gram-negative bacteria and yeast. Anal Biochem 2009, 394:192-201.

5. Bolten CJ, Kiefer P, Letisse F, Portais JC, Wittmann C: Sampling for metabolome analysis of microorganisms. Anal Chem 2007, 79:3843-3849.

6. Winder CL, Dunn WB, Schuler S, Broadhurst D, Jarvis R, Stephens GM, Goodacre R: Global metabolic profiling of Escherichia coli cultures: an evaluation of methods for quenching and extraction of intracellular metabolites. Anal Chem 2008, 80:2939-2948.

7. Katajamaa M, Miettinen J, Oresic M: MZmine: toolbox for processing and visualization of mass spectrometry based molecular profile data. Bioinformatics 2006, 22:634-636.

8. Katajamaa M, Oresic M: Processing methods for differential analysis of LC/MS profile data. BMC Bioinforma 2005, 6:179.

9. Smith CA, Want EJ, O'Maille G, Abagyan R, Siuzdak G: XCMS: processing mass spectrometry data for metabolite profiling using nonlinear peak alignment, matching, and identification. Anal Chem 2006, 78:779-787.

10. Behrends V, Tredwell GD, Bundy JG: A software complement to AMDIS for processing GC-MS metabolomic data. Anal Biochem 2011, 415:206-208.

11. Aggio R, Villas-Boas SG, Ruggiero K: Metab: an R package for highthroughput analysis of metabolomics data generated by GC-MS. Bioinformatics 2011, 27:2316-2318.

12. Hao J, Astle W, de-lorio M, Ebbels T: BATMAN-an R package for the automated quantification of metabolites from NMR spectra using a Bayesian Model. Bioinformatics 2011, 28:2088-2090.
13. Zheng C, Zhang S, Ragg S, Raftery D, Vitek O: Identification and quantification of metabolites in 1H NMR spectra by Bayesian model selection. Bioinformatics 2011, 27:1637-1644.

14. Muncey HJ, Jones R, De-lorio M, Ebbels TM: MetAssimulo: simulation of realistic NMR metabolic profiles. BMC Bioinforma 2010, 11:496.

15. Chagoyen M, Pazos F: MBRole: enrichment analysis of metabolomic data. Bioinformatics 2011, 27:730-731.

16. Cottret L, Wildridge D, Vinson F, Barrett MP, Charles H, Sagot MF, Jourdan F: MetExplore: a web server to link metabolomic experiments and genome-scale metabolic networks. Nucleic Acids Res 2010, 38:W132-W137.

17. Xia J, Wishart DS: MSEA: a web-based tool to identify biologically meaningful patterns in quantitative metabolomic data. Nucleic Acids Res 2010, 38:W71-W77.

18. Hans MA, Heinzle E, Wittmann C: Quantification of intracellular amino acids in batch cultures of Saccharomyces cerevisiae. Appl Microbiol Biotechnol 2001, 56:776-779.

19. Villas-Boas SG, Noel S, Lane GA, Attwood G, Cookson A: Extracellular metabolomics: A metabolic footprinting approach to assess fiber degradation in complex media. Anal Biochem 2006, 349:297-305.

20. Saez MJ, Lagunas R: Determination of Intermediary Metabolites in Yeast - Critical-Examination of Effect of Sampling Conditions and Recommendations for Obtaining True Levels. Mol Cell Biochem 1976, 13:73-78

21. Ewald JC, Heux S, Zamboni N: High-throughput quantitative metabolomics: workflow for cultivation, quenching, and analysis of yeast in a multiwell format. Anal Chem 2009, 81:3623-3629.

22. Wittmann C, Kromer JO, Kiefer P, Binz T, Heinzle E: Impact of the cold shock phenomenon on quantification of intracellular metabolites in bacteria. Anal Biochem 2004, 327:135-139.

23. Meyer $\mathrm{H}$, Liebeke M, Lalk M: A protocol for the investigation of the intracellular Staphylococcus aureus metabolome. Anal Biochem 2010, 401:250-259.

24. Atkinson DE: Energy Charge of Adenylate Pool as a Regulatory Parameter. Interaction with Feedback Modifiers. Biochemistry 1968, 7:4030-4034.

25. Taymaz-Nikerel H, de-Mey M, Ras C, ten-Pierick A, Seifar RM, van-Dam JC, Heijnen JJ, van-Gulik WM: Development and application of a differential method for reliable metabolome analysis in Escherichia coli. Anal Biochem 2009, 386:9-19.

26. Coulier L, Bas R, Jespersen S, Verheij E, van der-Werf MJ, Hankemeier T: Simultaneous quantitative analysis of metabolites using ion-pair liquid chromatography-electrospray ionization mass spectrometry. Anal Chem 2006, 78:6573-6582

27. Tredwell GD, Edwards-Jones B, Leak DJ, Bundy JG: The development of metabolomic sampling procedures for Pichia pastoris, and baseline metabolome data. PLOS One 2011, 6:e16286.

28. Faijes M, Mars AE, Smid EJ: Comparison of quenching and extraction methodologies for metabolome analysis of Lactobacillus plantarum. Microb Cell Fact 2007, 6(1):27.

29. T'Kindt R, Jankevics A, Scheltema RA, Zheng L, Watson DG, Dujardin JC, Breitling R, Coombs GH, Decuypere S: Towards an unbiased metabolic profiling of protozoan parasites: optimisation of a Leishmania sampling protocol for HILIC-orbitrap analysis. Anal Bioanal Chem 2010, 398:2059-2069

30. Yanes O, Tautenhahn R, Patti GJ, Siuzdak G: Expanding coverage of the metabolome for global metabolite profiling. Anal Chem 2011, 83:2152-2161.

31. Villas-Boas SG, Bruheim P: Cold glycerol-saline: the promising quenching solution for accurate intracellular metabolite analysis of microbial cells. Anal Biochem 2007, 370:87-97.

32. Soga T, Ohashi Y, Ueno Y, Naraoka H, Tomita M, Nishioka T: Quantitative metabolome analysis using capillary electrophoresis mass spectrometry. J Proteome Res 2003, 2:488-494.

33. Pieterse $B$, Jellema RH, van der-Werf MJ: Quenching of microbial samples for increased reliability of microarray data. J Microbiol Methods 2006, 64:207-216.

34. Liebeke M, Brozel VS, Hecker M, Lalk M: Chemical characterization of soil extract as growth media for the ecophysiological study of bacteria. Appl Microbiol Biotechnol 2009, 83:161-173.

35. Tautenhahn R, Patti GJ, Rinehart D, Siuzdak G: XCMS. A Web-Based Platform to Process Untargeted Metabolomic Data. Anal Chem: Online; 2012.

36. Schaub J, Schiesling C, Reuss M, Dauner M: Integrated sampling procedure for metabolome analysis. Biotechnol Prog 2006, 22:1434-1442. 
37. Watrous JD, Dorrestein PC: Imaging mass spectrometry in microbiology. Nat Rev Microbiol 2011, 9:683-694.

38. Wishart DS, Tzur D, Knox C, Eisner R, Guo AC, Young N, Cheng D, Jewell K, Arndt D, Sawhney S, et al: HMDB: the Human Metabolome Database. Nucleic Acids Res 2007, 35:D521-D526.

39. Jewison T, Knox C, Neveu V, Djoumbou Y, Guo AC, Lee J, Liu P, Mandal R, Krishnamurthy R, Sinelnikov I, et al: YMDB: the Yeast Metabolome Database. Nucleic Acids Res 2012, 40:D815-D820.

40. Smith CA, O'Maille G, Want EJ, Qin C, Trauger SA, Brandon TR, Custodio DE, Abagyan R, Siuzdak G: METLIN: a metabolite mass spectral database. Ther Drug Monit 2005, 27:747-751.

41. Botella E, Fogg M, Jules M, Piersma S, Doherty G, Hansen A, Denham EL, Le-Chat L, Veiga P, Bailey K, et al: pBaSysBioll: an integrative plasmid generating gfp transcriptional fusions for high-throughput analysis of gene expression in Bacillus subtilis. Microbiology 2010, 156:1600-1608.

42. Hochgrafe F, Wolf C, Fuchs S, Liebeke M, Lalk M, Engelmann S, Hecker M: Nitric oxide stress induces different responses but mediates comparable protein thiol protection in Bacillus subtilis and Staphylococcus aureus. J Bacteriol 2008, 190:4997-5008.

43. Liebeke M, Pother DC, Duy N, Albrecht D, Becher D, Hochgrafe F, Lalk M, Hecker $\mathrm{M}$, Antelmann H: Depletion of thiol-containing proteins in response to quinones in Bacillus subtilis. Mol Microbiol 2008, 69:1513-1529.

44. Bunk B, Kucklick M, Jonas R, Munch R, Schobert M, Jahn D, Hiller K: MetaQuant: a tool for the automatic quantification of GC/MS-based metabolome data. Bioinformatics 2006, 22:2962-2965.

45. Liebeke M, Meyer H, Donat S, Ohlsen K, Lalk M: A metabolomic view of Staphylococcus aureus and Its Ser/Thr kinase and phosphatase deletion mutants: involvement in cell wall biosynthesis. Chem Biol 2010, 17:820-830.

doi:10.1186/1475-2859-12-69

Cite this article as: Meyer et al: Methodological approaches to help unravel the intracellular metabolome of Bacillus subtilis. Microbial Cell Factories 2013 12:69.

\section{Submit your next manuscript to BioMed Central and take full advantage of:}

- Convenient online submission

- Thorough peer review

- No space constraints or color figure charges

- Immediate publication on acceptance

- Inclusion in PubMed, CAS, Scopus and Google Scholar

- Research which is freely available for redistribution 